\title{
Spirallike mappings and univalent subordination chains in $\mathbb{C}^{n}$
}

\author{
IAn Graham, Hidetaka Hamada, Gabriela KoHr and Mirela KoHr
}

\begin{abstract}
In this paper we consider non-normalized univalent subordination chains and the connection with the Loewner differential equation on the unit ball in $\mathbb{C}^{n}$. To this end, we study the most general form of the initial value problem for the transition mapping, and prove the existence and uniqueness of solutions. Also we introduce the notion of generalized spirallikeness with respect to a measurable matrix-valued mapping, and investigate this notion from the point of view of non-normalized univalent subordination chains. We prove that such a spirallike mapping can be imbedded as the first element of a univalent subordination chain, and we present various particular cases and examples. If the matrix-valued mapping is constant, we obtain the usual notion of spirallikeness with respect to a linear operator.
\end{abstract}

Mathematics Subject Classification (2000): 32H02 (primary); 30C45 (secondary).

\section{Introduction and preliminaries}

Let $\mathbb{C}^{n}$ denote the space of $n$ complex variables $z=\left(z_{1}, \ldots, z_{n}\right)$ with the Euclidean inner product $\langle z, w\rangle=\sum_{j=1}^{n} z_{j} \bar{w}_{j}$ and the Euclidean norm $\|z\|=\langle z, z\rangle^{1 / 2}$. The open ball $\left\{z \in \mathbb{C}^{n}:\|z\|<r\right\}$ is denoted by $B_{r}^{n}$ and the unit ball $B_{1}^{n}$ is denoted by $B^{n}$. The closed ball $\left\{z \in \mathbb{C}^{n}:\|z\| \leq r\right\}$ is denoted by $\bar{B}_{r}^{n}$. In the case of one complex variable, $B^{1}$ is denoted by $U$.

Let $L\left(\mathbb{C}^{n}, \mathbb{C}^{m}\right)$ be the space of linear and continuous operators from $\mathbb{C}^{n}$ into $\mathbb{C}^{m}$ with the standard operator norm and let $I_{n}$ be the identity in $L\left(\mathbb{C}^{n}, \mathbb{C}^{n}\right)$. If $\Omega$ is a domain in $\mathbb{C}^{n}$, let $H(\Omega)$ be the set of holomorphic mappings from $\Omega$ into $\mathbb{C}^{n}$. If $\Omega$ is a domain in $\mathbb{C}^{n}$ which contains the origin and $f \in H(\Omega)$, we say that $f$ is normalized if $f(0)=0$ and $D f(0)=I_{n}$. Let $S\left(B^{n}\right)$ be the set of normalized biholomorphic mappings on $B^{n}$. The set $S\left(B^{1}\right)$ is denoted by $S$. Also let $S^{*}\left(B^{n}\right)$ be the subset of $S\left(B^{n}\right)$ consisting of starlike mappings on $B^{n}$.

G. Kohr and M. Kohr are partially supported by the Romanian Ministry of Education and Research, UEFISCSU Grants PN-II-ID 524/2007 and 525/2007.

Received December 11, 2007; accepted June 18, 2008. 
If $f \in H\left(B^{n}\right)$, we say that $f$ is locally biholomorphic on $B^{n}$ if $J_{f}(z) \neq 0$, $z \in B^{n}$, where $J_{f}(z)=\operatorname{det} D f(z)$ and $D f(z)$ is the complex Jacobian matrix of $f$ at $z$. Let $\mathcal{L} S_{n}$ be the set of normalized locally biholomorphic mappings on $B^{n}$.

If $f, g \in H\left(B^{n}\right)$, we say that $f$ is subordinate to $g(f \prec g)$ if there exists a Schwarz mapping $v$ (i.e. $v \in H\left(B^{n}\right)$ and $\|v(z)\| \leq\|z\|, z \in B^{n}$ ) such that $f=g \circ v$.

Definition 1.1. A mapping $f: B^{n} \times[0, \infty) \rightarrow \mathbb{C}^{n}$ is called a univalent subordination chain if $f(\cdot, t)$ is biholomorphic on $B^{n}, f(0, t)=0$ for $t \geq 0$, and $f(\cdot, s) \prec f(\cdot, t), 0 \leq s \leq t<\infty$.

The above subordination condition is equivalent to the existence of a unique Schwarz mapping $v=v(z, s, t)$, called the transition mapping associated with $f(z, t)$, such that $f(z, s)=f(v(z, s, t), t), z \in B^{n}, t \geq s \geq 0$.

If $f(z, t)$ is a univalent subordination chain such that $D f(0, t)=e^{t} I_{n}$ for $t \geq$ 0 , we say that $f(z, t)$ is a normalized univalent subordination chain or a Loewner chain.

The following class of mappings in $H\left(B^{n}\right)$ is important in our treatment:

$$
\mathcal{N}=\left\{h \in H\left(B^{n}\right): h(0)=0, \Re\langle h(z), z\rangle>0, z \in B^{n} \backslash\{0\}\right\} .
$$

Several applications of this class in the study of biholomorphic mappings and univalent subordination chains on the unit ball in $\mathbb{C}^{n}$ may be found in $[6,9,11,19,26$, 29, 30].

For a given operator $A \in L\left(\mathbb{C}^{n}, \mathbb{C}^{n}\right)$, let us denote by

$$
m(A)=\min \{\Re\langle A(z), z\rangle:\|z\|=1\} \text { and } k(A)=\max \{\Re\langle A(z), z\rangle:\|z\|=1\} .
$$

Also let $|V(A)|=\max _{\|z\|=1}|\langle A(z), z\rangle|$ be the numerical radius of the operator $A$. Then $|V(A)| \leq\|A\| \leq 2|V(A)|$ by [13, Theorem 1.3.1] (see also [1, Theorem 4.1]; $c f$. $[16,17])$. Further, let $k_{+}(A)=\lim _{t \rightarrow \infty} \frac{\ln \left\|e^{t A}\right\|}{t}$. It is well known that this limit exists, and for each $\omega>k_{+}(A)$, there exists $\delta=\delta(\omega)>0$ such that $\left\|e^{t A}\right\| \leq \delta e^{\omega t}$, $t \geq 0$. In addition, $k_{+}(A)=\max \{\Re \lambda: \lambda \in \sigma(A)\}$ where $\sigma(A)$ is the spectrum of $A$ (see [3, Theorem 4.1], [4]).

To prove our results, we need the following lemma (see [8, Lemma 1.2]; $c f$. [6, Theorem 1.2]).

Lemma 1.2. Let $h: B^{n} \rightarrow \mathbb{C}^{n}$ be a mapping such that $h \in \mathcal{N}, D h(0)=A$ and $m(A)>0$. Then

$$
\|h(z)\| \leq \frac{4 r}{(1-r)^{2}}|V(A)|,\|z\| \leq r<1 .
$$

Definition 1.3. ( $c f .[12,30])$ Let $A \in L\left(\mathbb{C}^{n}, \mathbb{C}^{n}\right)$ be such that $m(A)>0$. Also let $\Omega$ be a domain in $\mathbb{C}^{n}$ which contains the origin. We say that $\Omega$ is spirallike with respect to $A$ if $e^{-t A}(w) \in \Omega$, for all $w \in \Omega$ and $t \geq 0$.

A mapping $f \in S\left(B^{n}\right)$ is called spirallike with respect to $A$ if $f\left(B^{n}\right)$ is a spirallike domain with respect to $A$. 


\section{Remark 1.4.}

(i) It is well known that a mapping $f \in \mathcal{L} S_{n}$ is spirallike with respect to $A$ if and only if $\Re\left\langle[D f(z)]^{-1} A f(z), z\right\rangle>0, z \in B^{n} \backslash\{0\}$ (see [30]).

Other results related to spirallike mappings with respect to a linear operator can be found in [5, 12, 26, 27].

(ii) A mapping $f$ is called spirallike of type $\alpha \in(-\pi / 2, \pi / 2)$ if $f$ is spirallike with respect to $A=e^{-i \alpha} I_{n}$ (see [14]; $c f$. [30]). Hence $f \in S^{*}\left(B^{n}\right)$ if and only if $f$ is spirallike of type zero.

Hamada and Kohr [14] proved that any spirallike mapping $f$ of type $\alpha$ can be imbedded as the first element of the Loewner chain $f(z, t)=e^{(1-i a) t} f\left(e^{i a t} z\right)$, where $a=\tan \alpha$. This Loewner chain has the property that $\left\{e^{-t} f(\cdot, t)\right\}_{t \geq 0}$ is a normal family on $B^{n}$. However, if $f$ is a spirallike mapping with respect to a given linear operator $A$, then $f$ need not be imbedded in a Loewner chain $f(z, t)$ such that $\left\{e^{-t} f(\cdot, t)\right\}_{t \geq 0}$ is a normal family on $B^{n}$. On the other hand, if $f$ is spirallike with respect to $A$, then $f(z, t)=e^{t A} f(z)$ is a (non-normalized) univalent subordination chain (see [8]).

We next introduce the notion of generalized parametric representation with respect to a measurable mapping $A:[0, \infty) \rightarrow L\left(\mathbb{C}^{n}, \mathbb{C}^{n}\right)$.

Definition 1.5. Let $A:[0, \infty) \rightarrow L\left(\mathbb{C}^{n}, \mathbb{C}^{n}\right)$ be a measurable mapping such that $m(A(t))>0$ for $t \geq 0$ and $\int_{0}^{\infty} m(A(t)) d t=\infty$. Moreover, assume that $\|A(\cdot)\|$ is uniformly bounded on $[0, \infty)$ and

$$
\int_{s}^{t} A(\tau) d \tau \circ \int_{r}^{s} A(\tau) d \tau=\int_{r}^{s} A(\tau) d \tau \circ \int_{s}^{t} A(\tau) d \tau, t \geq s \geq r \geq 0 .
$$

Also, let $f \in H\left(B^{n}\right)$ be a normalized mapping. We say that $f$ has generalized parametric representation with respect to $A$ (and we denote the class of such $f$ by $\left.\tilde{S}_{A}^{0}\left(B^{n}\right)\right)$ if there exists a mapping $h: B^{n} \times[0, \infty) \rightarrow \mathbb{C}^{n}$ such that $h(\cdot, t) \in \mathcal{N}$, $D h(0, t)=A(t)$ for $t \in[0, \infty), h(z, \cdot)$ is measurable on $[0, \infty)$ for $z \in B^{n}$, and

$$
f(z)=\lim _{t \rightarrow \infty} e^{\int_{0}^{t} A(\tau) d \tau} v(z, t)
$$

locally uniformly on $B^{n}$, where $v=v(z, t)$ is the unique locally absolutely continuous solution on $[0, \infty)$ of the initial value problem

$$
\frac{\partial v}{\partial t}=-h(v, t) \quad \text { a.e. } t \geq 0, v(z, 0)=z
$$

for all $z \in B^{n}$. 


\section{Remark 1.6.}

(i) The condition (1.1) is satisfied if $A(t)$ is constant or if $A(t)$ is diagonal for $t \geq 0$. Note that the condition (1.1) implies the following equality:

$$
A(t) \circ \int_{0}^{t} A(\tau) d \tau=\int_{0}^{t} A(\tau) d \tau \circ A(t) \text {, a.e. } t \in[0, \infty) .
$$

Indeed, since $\|A(\cdot)\|$ is uniformly bounded, we have

$$
\lim _{\varepsilon \rightarrow 0} \frac{1}{\varepsilon} \int_{t}^{t+\varepsilon} A(\tau) d \tau=A(t) \text { a.e. } t \geq s
$$

Next, let $s=t, r=0$, and replace $t$ by $t+\varepsilon$ in (1.1). Then

$$
\frac{1}{\varepsilon} \int_{t}^{t+\varepsilon} A(\tau) d \tau \circ \int_{0}^{t} A(\tau) d \tau=\int_{0}^{t} A(\tau) d \tau \circ \frac{1}{\varepsilon} \int_{t}^{t+\varepsilon} A(\tau) d \tau .
$$

Letting $\varepsilon \rightarrow 0$ in the above equality, we obtain (1.4), as desired.

(ii) The existence and uniqueness of the locally absolutely continuous solution $v(z, t)$ to the initial value problem (1.3) follows from Theorem 2.1. Moreover, we shall see that the limit in (1.2) exists if the condition (2.6) holds.

(iii) If $A(t) \equiv A \in L\left(\mathbb{C}^{n}, \mathbb{C}^{n}\right)$ with $m(A)>0$ in Definition 1.5 , we obtain the class of mappings which have $A$-parametric representation. This class has been recently introduced in [8]. Further, if $A(t) \equiv I_{n}$, we obtain the usual class $S^{0}\left(B^{n}\right)$ of mappings which have parametric representation. A number of properties of mappings which have parametric representation were obtained in $[6,7,9,11,18]$. Poreda ( $[24,25])$ originally introduced the notion of parametric representation on the unit polydisc in $\mathbb{C}^{n}$ and Kohr [18] introduced this notion on the Euclidean unit ball in $\mathbb{C}^{n}$. If $n=1, S^{0}(U)=S$ (see [22]), but $S^{0}\left(B^{n}\right) \varsubsetneqq S\left(B^{n}\right)$ for $n \geq 2$ (see [6]).

(iv) One of the most important properties of mappings which have parametric representation is that a normalized mapping $f \in H\left(B^{n}\right)$ belongs to $S^{0}\left(B^{n}\right)$ if and only if $f$ can be imbedded as the first element of a Loewner chain $f(z, t)$ such that $\left\{e^{-t} f(\cdot, t)\right\}_{t \geq 0}$ is a normal family on $B^{n}$ (see $[6,9,11] ; c f$. [24]).

(v) Let $A:[0, \infty) \rightarrow L\left(\mathbb{C}^{n}, \mathbb{C}^{n}\right)$ be a measurable mapping, which satisfies the assumptions of Definition 1.5. Then

$$
e^{\int_{0}^{t} m(A(\tau)) d \tau} \leq\left\|e^{\int_{0}^{t} A(\tau) d \tau}\right\| \leq e^{\int_{0}^{t} k(A(\tau)) d \tau}, t \in[0, \infty)
$$

and

$$
e^{-\int_{0}^{t} k(A(\tau)) d \tau} \leq\left\|e^{-\int_{0}^{t} A(\tau) d \tau}\right\| \leq e^{-\int_{0}^{t} m(A(\tau)) d \tau}, t \in[0, \infty) .
$$

Hence $\lim _{t \rightarrow \infty}\left\|e^{\int_{0}^{t} A(\tau) d \tau}\right\|=\infty$. 
Proof. Indeed, fix $u \in \mathbb{C}^{n}$ with $\|u\|=1$, and let $q_{u}(t)=\left\|e^{\int_{0}^{t} A(\tau) d \tau}(u)\right\|^{2}$ for $t \geq 0$. Then $q_{u}$ is a locally Lipschitz continuous function on $[0, \infty)$, since $\|A(\cdot)\|$ is uniformly bounded on $[0, \infty)$. Hence $q_{u}(t)$ is differentiable for almost all $t \in$ $[0, \infty)$. In view of $(1.4)$, we deduce that

$$
q_{u}^{\prime}(t)=2 \Re\left\langle A(t) e^{\int_{0}^{t} A(\tau) d \tau}(u), e^{\int_{0}^{t} A(\tau) d \tau}(u)\right\rangle, \text { a.e. } t \geq 0
$$

Hence

$$
2 m(A(t)) q_{u}(t) \leq q_{u}^{\prime}(t) \leq 2 k(A(t)) q_{u}(t), \text { a.e. } t \geq 0,
$$

i.e.

$$
2 m(A(t)) \leq \frac{q_{u}^{\prime}(t)}{q_{u}(t)} \leq 2 k(A(t)), \text { a.e. } t \geq 0 .
$$

Integrating both sides of the above inequality, we easily obtain the relation (1.5). The relation $\lim _{t \rightarrow \infty}\left\|e^{\int_{0}^{t} A(\tau) d \tau}\right\|=\infty$ is a direct consequence of (1.5) and the fact that $\int_{0}^{\infty} m(A(\tau)) d \tau=\infty$. To deduce (1.6), it suffices to apply an argument similar to that used for (1.5).

In this paper we investigate the connection between generalized parametric representation with respect to a measurable mapping $A:[0, \infty) \rightarrow L\left(\mathbb{C}^{n}, \mathbb{C}^{n}\right)$ with $m(A(t))>0, t \geq 0$, and univalent subordination chains. Also, we introduce the notion of generalized spirallikeness and study this notion from the point of view of non-normalized univalent subordination chains. Finally we obtain various examples of generalized spirallike mappings with respect to measurable diagonal matrices.

\section{Parametric representation with respect to a measurable operator and univalent subordination chains}

In this section we study the existence of solutions to the initial value problem (2.1) in the most general form, i.e. when the mapping $h=h(\cdot, t) \in \mathcal{N}$ is such that $D h(0, t)=A(t) \in L\left(\mathbb{C}^{n}, \mathbb{C}^{n}\right)$ for $t \geq 0$. Particular cases of Theorem 2.1 were obtained in [19, Theorem 2.1] (in the case that $D h(0, t)=I_{n}$ for $t \geq 0$ ), [26, Lemma 4.1] (in the case that $h(\cdot, t)=q \in \mathcal{N}$ for $t \geq 0$ ) and [8, Theorem 2.1] (in the case that $D h(0, t)=A \in L\left(\mathbb{C}^{n}, \mathbb{C}^{n}\right)$ for $t \geq 0$, where $\left.m(A)>0\right)$.

Theorem 2.1. Let $A:[0, \infty) \rightarrow L\left(\mathbb{C}^{n}, \mathbb{C}^{n}\right)$ be a measurable mapping which satisfies the assumptions of Definition 1.5. Also let $h=h(z, t): B^{n} \times[0, \infty) \rightarrow \mathbb{C}^{n}$ be a mapping which satisfies the following conditions:

(i) $h(\cdot, t) \in \mathcal{N}, D h(0, t)=A(t)$ for $t \geq 0$;

(ii) $h(z, \cdot)$ is measurable on $[0, \infty)$ for $z \in B^{n}$. 
Then for each $s \geq 0$ and $z \in B^{n}$, the initial value problem

$$
\frac{\partial v}{\partial t}=-h(v, t) \text { a.e. } t \geq s, v(z, s, s)=z
$$

has a unique solution $v=v(z, s, t)$ such that $v(\cdot, s, t)$ is a univalent Schwarz mapping, $v(z, s, \cdot)$ is Lipschitz continuous on $[s, \infty)$ locally uniformly with respect to $z \in B^{n}, D v(0, s, t)=\exp \left(-\int_{s}^{t} A(\tau) d \tau\right)$ for $t \geq s \geq 0$, and the following relations hold:

$$
\begin{gathered}
\frac{\|v(z, s, t)\|}{(1-\|v(z, s, t)\|)^{2}} \leq e^{-\int_{s}^{t} m(A(\tau)) d \tau} \frac{\|z\|}{(1-\|z\|)^{2}}, z \in B^{n}, t \geq s \geq 0 . \\
\frac{\|v(z, s, t)\|}{(1+\|v(z, s, t)\|)^{2}} \geq e^{-\int_{s}^{t} k(A(\tau)) d \tau} \frac{\|z\|}{(1+\|z\|)^{2}}, z \in B^{n}, t \geq s \geq 0 .
\end{gathered}
$$

Proof. As in the proof of [19, Theorem 2.1] (see also the proof of [8, Theorem 2.1]), we may apply the classical method of successive approximations to construct the unique locally Lipschitz continuous solution on $[s, \infty)$ to the initial value problem (2.1) (which in this case is globally Lipschitz). We omit the details.

We only prove that $D v(0, s, t)=\exp \left(-\int_{s}^{t} A(\tau) d \tau\right)$. To this end, fix $s \geq 0$ and let $V(t)=D v(0, s, t)$ for $t \geq s$. By using the Cauchy integral formula and the Lipschitz continuity of $v(z, s, \cdot)$ on $[s, \infty)$ locally uniformly with respect to $z \in B^{n}$, we deduce that for each $r \in(0,1)$, there exists a constant $L=L(r)>0$ such that

$$
\left\|V\left(t_{1}\right)-V\left(t_{2}\right)\right\| \leq L\left|t_{1}-t_{2}\right|, t_{1}, t_{2} \in[s, \infty) .
$$

Thus $V$ is Lipschitz continuous on $[s, \infty)$, and hence $\frac{\partial V}{\partial t}$ exists a.e. $t \geq s$. Moreover, since $D h(0, t)=A(t)$ and $v(z, s, t)$ is the solution of (2.1), we deduce that $V$ satisfies the initial value problem

$$
\frac{\partial V}{\partial t}=-A(t) V(t) \text { a.e. } t \geq s, V(s)=I_{n} .
$$

Taking into account the condition (1.1) and solving the above initial value problem, we obtain the unique Lipschitz continuous solution $V(t)=\exp \left(-\int_{s}^{t} A(\tau) d \tau\right)$ on $[s, \infty)$ (compare with [4, page 564], [2] and [31, page 431]). Indeed, since $\|A(t)\|$ is bounded, we have

$$
\lim _{\varepsilon \rightarrow 0} \frac{1}{\varepsilon} \int_{t}^{t+\varepsilon} A(\tau) d \tau=A(t) \quad \text { a.e. } t \geq s .
$$

Let $t \geq s$ be fixed such that the above equality holds. Then there exists $M(\varepsilon)$ such that

$$
\frac{1}{\varepsilon} \int_{t}^{t+\varepsilon} A(\tau) d \tau=A(t)+M(\varepsilon)
$$


and $M(\varepsilon) \rightarrow 0$ as $\varepsilon \rightarrow 0$. Therefore, we obtain that

$$
\begin{aligned}
V^{\prime}(t) & =\lim _{\varepsilon \rightarrow 0} \frac{1}{\varepsilon}\{V(t+\varepsilon)-V(t)\} \\
& =\lim _{\varepsilon \rightarrow 0} \frac{1}{\varepsilon}\left\{\exp \left(-\int_{s}^{t+\varepsilon} A(\tau) d \tau\right)-\exp \left(-\int_{s}^{t} A(\tau) d \tau\right)\right\} \\
& =\lim _{\varepsilon \rightarrow 0} \frac{1}{\varepsilon}\left\{\exp \left(-\int_{t}^{t+\varepsilon} A(\tau) d \tau\right)-I_{n}\right\} V(t) \\
& =\lim _{\varepsilon \rightarrow 0}\{-(A(t)+M(\varepsilon))+\varepsilon \cdot \text { (bounded) }\} V(t)=-A(t) V(t) .
\end{aligned}
$$

Finally, it suffices to use arguments similar to those in the proofs of [19, Lemma 2.2] and [12, Lemma 2], to deduce (2.2) and (2.3). Indeed, since $h(\cdot, t) \in \mathcal{N}$ and $D h(0, t)=A(t)$, we deduce as in the proof of [19, Lemma 2.1] that

$$
\Re\langle A(t)(z), z\rangle \frac{1-\|z\|}{1+\|z\|} \leq \Re\langle h(z, t), z\rangle \leq \Re\langle A(t)(z), z\rangle \frac{1+\|z\|}{1-\|z\|},
$$

for all $z \in B^{n}$ and $t \geq 0$. Therefore we obtain that

$$
m(A(t))\|z\|^{2} \frac{1-\|z\|}{1+\|z\|} \leq \Re\langle h(z, t), z\rangle \leq k(A(t))\|z\|^{2} \frac{1+\|z\|}{1-\|z\|}, z \in B^{n}, t \geq 0 .
$$

Next fix $z \in B^{n} \backslash\{0\}$ and $s \geq 0$. Then $v(z, s, t) \neq 0$ and from (2.4) we obtain that

$$
\frac{d\|v(t)\|}{d t}=-\frac{1}{\|v(t)\|} \Re\langle h(v(t), t), v(t)\rangle \leq-m(A(t))\|v(t)\| \frac{1-\|v(t)\|}{1+\|v(t)\|}, \text { a.e. } t \geq s .
$$

Therefore, we obtain that

$$
\frac{1+\|v(t)\|}{(1-\|v(t)\|)\|v(t)\|} \frac{d\|v(t)\|}{d t} \leq-m(A(t)), \text { a.e. } t \geq s .
$$

Since $\|v(z, s, \cdot)\|$ is locally Lipschitz continuous on $[s, \infty), m(A(\cdot))$ and $k(A(\cdot))$ are measurable functions, we may integrate both sides of the above inequality and change variables, to deduce (2.2), as claimed. The relation (2.3) follows in a similar way to (2.2). This completes the proof.

We next prove that the Lipschitz continuous solution of the initial value problem (2.1) generates a non-normalized univalent subordination chain, if the condition (2.6) holds (see [26] in the case $A(t) \equiv I_{n} ; c f$. [9, Theorem 8.1.5], [8, Theorem 2.3]). 


\section{Remark 2.2.}

(i) The condition (2.6) is satisfied if there exists $\eta>0$ such that

$$
\|A(t)\|-2 m(A(t)) \leq-\eta, t \geq 0 .
$$

Indeed, since

$$
\begin{aligned}
\int_{s}^{\infty}\left\|e^{\int_{s}^{t}\left[A(\tau)-2 m(A(\tau)) I_{n}\right] d \tau}\right\| d t & \leq \int_{s}^{\infty} e^{\int_{s}^{t}[\|A(\tau)\|-2 m(A(\tau))] d \tau} d t \\
& \leq \int_{s}^{\infty} e^{-\eta(t-s)} d t=\frac{1}{\eta}, s \in[0, \infty)
\end{aligned}
$$

the conclusion follows.

(ii) If $A(t) \equiv A \in L\left(\mathbb{C}^{n}, \mathbb{C}^{n}\right)$ then the condition (2.6) is equivalent to

$$
\int_{0}^{\infty}\left\|e^{\left(A-2 m(A) I_{n}\right) t}\right\| d t<\infty
$$

and this relation is satisfied if $k_{+}(A)<2 m(A)$ (see [8]).

(iii) If $A(t)$ is diagonal with eigenvalues $a_{1}(t), \ldots, a_{n}(t)$ for $t \geq 0$, then the condition (2.6) is satisfied if there exists $\delta>0$ such that

$$
\max \left\{\Re a_{1}(t), \ldots, \Re a_{n}(t)\right\}-2 \min \left\{\Re a_{1}(t), \ldots, \Re a_{n}(t)\right\} \leq-\delta, t \geq 0
$$

Indeed, elementary computations yield that

$$
\begin{aligned}
& \int_{s}^{\infty}\left\|e^{\int_{s}^{t}\left[A(\tau)-2 m(A(\tau)) I_{n}\right] d \tau}\right\| d t \\
& \leq \int_{s}^{\infty} e^{\int_{s}^{t}\left[\max \left\{\Re a_{1}(\tau), \ldots, \Re a_{n}(\tau)\right\}-2 \min \left\{\Re a_{1}(\tau), \ldots, \Re a_{n}(\tau)\right\}\right] d \tau} d t \\
& \leq \int_{s}^{\infty} e^{-\delta(t-s)} d t=\frac{1}{\delta}
\end{aligned}
$$

for $s \geq 0$, and hence

$$
\sup _{s \geq 0} \int_{s}^{\infty}\left\|e^{\int_{s}^{t}\left[A(\tau)-2 m(A(\tau)) I_{n}\right] d \tau}\right\| d t \leq \frac{1}{\delta} .
$$

Note that the condition (2.5) is equivalent to

$$
k_{+}(A(t))-2 m(A(t)) \leq-\delta, t \geq 0 .
$$

It would be interesting to see whether the above condition implies (2.6). 
Theorem 2.3. Let $h=h(z, t): B^{n} \times[0, \infty) \rightarrow \mathbb{C}^{n}$ and $A:[0, \infty) \rightarrow L\left(\mathbb{C}^{n}, \mathbb{C}^{n}\right)$ satisfy the assumptions of Theorem 2.1 and let $v=v(z, s, t)$ be the Lipschitz continuous solution on $[s, \infty)$ of the initial value problem (2.1). Also, assume that the following condition holds:

$$
\sup _{s \geq 0} \int_{s}^{\infty}\left\|e^{\int_{s}^{t}\left[A(\tau)-2 m(A(\tau)) I_{n}\right] d \tau}\right\| d t<\infty
$$

Then the limit

$$
\lim _{t \rightarrow \infty} e^{\int_{0}^{t} A(\tau) d \tau} v(z, s, t)=f(z, s)
$$

exists locally uniformly on $B^{n}$ for each $s \geq 0$. Moreover, $f(z, t)$ is a univalent subordination chain such that $D f(0, t)=e^{\int_{0}^{t} A(\tau) d \tau},\left\{e^{-\int_{0}^{t} A(\tau) d \tau} f(\cdot, t)\right\}_{t \geq 0}$ is a normal family on $B^{n}$ and $f=f(\cdot, 0)$ has generalized parametric representation with respect to $A$.

Proof. We use arguments similar to those in the proof of [8, Theorem 2.3] and [26, Lemma 4.2]. Fix $s \geq 0$ and let $u(z, s, t)=e^{\int_{0}^{t} A(\tau) d \tau} v(z, s, t)$ for $z \in B^{n}$ and $t \geq s$. Also let $g(z, t)=h(z, t)-A(t)(z)$ for $z \in B^{n}$ and $t \geq 0$. Then $g(\cdot, t) \in H\left(\bar{B}^{n}\right)$, $g(0, t)=0$ and $\operatorname{Dg}(0, t)=0$.

We first prove that $u(z, s, \cdot)$ is locally Lipschitz continuous on $[s, \infty)$ locally uniformly with respect to $z \in B^{n}$.

Fix $s \geq 0, T>s$ and $r \in(0,1)$. Also let $t_{1}, t_{2} \in[s, T]$. Then

$$
\begin{aligned}
& \left\|u\left(z, s, t_{1}\right)-u\left(z, s, t_{2}\right)\right\| \\
& \leq e^{\int_{0}^{t_{1}}\|A(\tau)\| d \tau}\left\|v\left(z, s, t_{1}\right)-v\left(z, s, t_{2}\right)\right\|+\left\|v\left(z, s, t_{2}\right)\right\|\left\|e^{\int_{0}^{t_{1}} A(\tau) d \tau}-e^{\int_{0}^{t_{2}} A(\tau) d \tau}\right\| .
\end{aligned}
$$

By hypothesis, there exists $b>0$ such that $\|A(t)\| \leq b$ for $t \geq 0$. Also, since $v(z, s, \cdot)$ is Lipschitz continuous on $[s, \infty)$ locally uniformly with respect to $z \in$ $B^{n}$, we deduce in view of the above relations that there exists $N=N(r, b)>0$ such that

$$
\left\|u\left(z, s, t_{1}\right)-u\left(z, s, t_{2}\right)\right\| \leq e^{b T} N(r, b)\left|t_{1}-t_{2}\right|+r\left\|e^{C\left(t_{1}\right)}-e^{C\left(t_{2}\right)}\right\|,
$$

where $C(t)=\int_{0}^{t} A(\tau) d \tau$ for $t \geq s$. Since

$$
\left\|C\left(t_{1}\right)-C\left(t_{2}\right)\right\| \leq b\left|t_{1}-t_{2}\right|,
$$

we deduce that the mapping $C$ is Lipschitz continuous on $[0, \infty)$. Since the mapping $q(t)=e^{C(t)}$ is locally Lipschitz continuous for $t \geq 0$ and $q^{\prime}(t)=A(t) e^{C(t)}$ for almost all $t \geq 0$, we deduce that

$$
\left\|e^{C\left(t_{1}\right)}-e^{C\left(t_{2}\right)}\right\| \leq \max _{t \in[s, T]}\left\|A(t) e^{C(t)}\right\| \cdot\left|t_{1}-t_{2}\right| \leq b e^{b T}\left|t_{1}-t_{2}\right|, t_{1}, t_{2} \in[s, T] .
$$


Taking into account the above relation and (2.8), we deduce that $\left\|u\left(z, s, t_{1}\right)-u\left(z, s, t_{2}\right)\right\| \leq e^{b T} N(r, b)\left|t_{1}-t_{2}\right|+b r e^{b T}\left|t_{1}-t_{2}\right|=N^{*}(r, b, T)\left|t_{1}-t_{2}\right|$.

Thus $u(z, s, \cdot)$ is Lipschitz continuous on $[s, T]$ locally uniformly with respect to $z \in B^{n}$, and hence $\frac{\partial u}{\partial t}(z, s, t)$ exists for almost all $t \in[s, T]$ and for all $z \in B^{n}$.

Using the relation (2.1), it is not difficult to deduce that $u(t)=u(z, s, t)$ satisfies the following relation:

$$
\frac{\partial u}{\partial t}=-e^{\int_{0}^{t} A(\tau) d \tau} g\left(e^{-\int_{0}^{t} A(\tau) d \tau} u, t\right) \text { a.e. } t \geq s .
$$

We next prove that the limit in (2.7) exists locally uniformly on $B^{n}$. In view of the local Lipschitz continuity of $u(z, s, \cdot)$ on $[s, \infty)$ locally uniformly with respect to $z \in B^{n}$ and the relation (2.9), we deduce that

$$
\left\|u\left(z, s, t_{1}\right)-u\left(z, s, t_{2}\right)\right\| \leq \int_{t_{1}}^{t_{2}}\left\|e^{\int_{0}^{t} A(\tau) d \tau}\right\| \cdot\left\|g\left(e^{-\int_{0}^{t} A(\tau) d \tau} u(z, s, t), t\right)\right\| d t .
$$

In view of Lemma 1.2 and the fact that $|V(A(t))| \leq\|A(t)\| \leq b$ for $t \geq 0$, we deduce that

$$
\|h(z, t)\| \leq \frac{4 r}{(1-r)^{2}}|V(A(t))| \leq \frac{4 r b}{(1-r)^{2}}=K(r, b),
$$

for $\|z\| \leq r<1$ and $t \geq 0$.

Moreover, using (2.11) and the relations $g(0, t)=0, D g(0, t)=0$, and

$$
\|g(z, t)\| \leq\|h(z, t)\|+\|A(t)(z)\| \leq K(r, b)+r b=K^{*}(r, b)
$$

for $\|z\| \leq r<1$ and $t \geq 0$, we obtain in view of the Schwarz lemma that

$$
\|g(z, t)\| \leq \frac{K^{*}(r, b)}{r^{2}}\|z\|^{2},\|z\| \leq r<1, t \geq 0 .
$$

In view of the above inequality, the relations (2.2) and (2.10), we deduce that

$$
\begin{aligned}
\left\|u\left(z, s, t_{1}\right)-u\left(z, s, t_{2}\right)\right\| & \leq \int_{t_{1}}^{t_{2}}\left\|e_{0}^{\int_{0}^{t} A(\tau) d \tau}\right\| \cdot \frac{K^{*}(r, b)}{r^{2}}\|v(z, s, t)\|^{2} d t \\
& \leq \frac{K^{*}(r, b)}{(1-r)^{4}} \int_{t_{1}}^{t_{2}}\left\|e^{\int_{0}^{t} A(\tau) d \tau}\right\| e^{-2 \int_{s}^{t} m(A(\tau)) d \tau} d t \\
& \leq \frac{K^{*}(r, b)}{(1-r)^{4}} e^{2 \int_{0}^{s} m(A(\tau)) d \tau} \int_{t_{1}}^{t_{2}}\left\|e^{\int_{0}^{t}\left[A(\tau)-2 m(A(\tau)) I_{n}\right] d \tau}\right\| d t .
\end{aligned}
$$

Since the integral $\int_{0}^{\infty}\left\|e^{t_{0}^{t}\left[A(\tau)-2 m(A(\tau)) I_{n}\right] d \tau}\right\| d t$ is convergent in view of (2.6), we deduce from the above inequality that for each $\varepsilon>0$, there exists $t_{0}^{*}=t_{0}^{*}(r, b, s)>$ 0 such that

$$
\left\|u\left(z, s, t_{1}\right)-u\left(z, s, t_{2}\right)\right\|<\varepsilon,\|z\| \leq r<1, t_{2} \geq t_{1} \geq t_{0}^{*} .
$$


Using the Weierstrass theorem, we deduce that the limit (2.7) exists locally uniformly on $B^{n}$, as desired.

Finally we prove that $f(z, t)$ given by $(2.7)$ is a univalent subordination chain such that $\left\{e^{-\int_{0}^{t} A(\tau) d \tau} f(\cdot, t)\right\}_{t \geq 0}$ is a normal family on $B^{n}$. Clearly $f(0, t)=0$. Taking into account the condition (1.1) and the fact that $D v(0, s, t)=e^{-\int_{s}^{t} A(\tau) d \tau}$, we deduce that $D f(0, t)=e^{\int_{0}^{t} A(\tau) d \tau}$ for $t \geq 0$. Hence $f(\cdot, t)$ is a biholomorphic mapping on $B^{n}$ for $t \geq 0$, by Hurwitz's theorem. Moreover, in view of uniqueness of solutions to the initial value problem (2.1), we deduce that $v(z, s, t)$ satisfies the semigroup property

$$
v(z, s, t)=v(v(z, s, \lambda), \lambda, t), 0 \leq s \leq \lambda \leq t<\infty .
$$

Then (2.7) and the above equality yield that

$$
\begin{aligned}
f(z, s) & =\lim _{t \rightarrow \infty} e^{\int_{0}^{t} A(\tau) d \tau} v(z, s, t) \\
& =\lim _{t \rightarrow \infty} e^{\int_{0}^{t} A(\tau) d \tau} v(v(z, s, \lambda), \lambda, t)=f(v(z, s, \lambda), \lambda) .
\end{aligned}
$$

Concluding the above arguments, we deduce that $f(z, t)$ is a univalent subordination chain, as claimed.

On the other hand, as in the above proof and using the condition (1.1), we deduce that for each $r \in(0,1)$ there exists $K^{*}(r, b)>0$ such that for $s \leq t_{1}<$ $t_{2}<\infty$,

$$
\begin{aligned}
& \left\|e^{-\int_{0}^{s} A(\tau) d \tau} u\left(z, s, t_{1}\right)-e^{-\int_{0}^{s} A(\tau) d \tau} u\left(z, s, t_{2}\right)\right\| \\
& \leq \frac{K^{*}(r, b)}{(1-r)^{4}} \int_{t_{1}}^{t_{2}}\left\|e^{\int_{s}^{t}\left[A(\tau)-2 m(A(\tau)) I_{n}\right] d \tau}\right\| d t \\
& \leq \frac{K^{*}(r, b)}{(1-r)^{4}} \cdot \sup _{x \geq 0} \int_{x}^{\infty}\left\|e^{\int_{x}^{t}\left[A(\tau)-2 m(A(\tau)) I_{n}\right] d \tau}\right\| d t=L^{*}(r, A)<\infty,\|z\| \leq r .
\end{aligned}
$$

Since $\lim _{t \rightarrow \infty} e^{-\int_{0}^{s} A(\tau) d \tau} u(z, s, t)=e^{-\int_{0}^{s} A(\tau) d \tau} f(z, s)$, we obtain in view of the above relation that

$$
\begin{aligned}
& \left\|e^{-\int_{0}^{s} A(\tau) d \tau} f(z, s)\right\| \leq L^{*}(r, A)+\left\|e^{-\int_{0}^{s} A(\tau) d \tau} u(z, s, s)\right\| \\
& \leq L^{*}(r, A)+r,\|z\| \leq r<1
\end{aligned}
$$

Hence $\left\{e^{-\int_{0}^{s} A(\tau) d \tau} f(\cdot, s)\right\}_{s \geq 0}$ is a normal family on $B^{n}$, as desired.

In view of Theorems 2.1 and 2.3, Definition 1.5 and Remark 1.6, we obtain the following consequence, which yields that any mapping which has generalized parametric representation with respect to a measurable linear operator can be imbedded in a (non-normalized) univalent subordination chain. If $A(t) \equiv A \in L\left(\mathbb{C}^{n}, \mathbb{C}^{n}\right)$, we obtain [8, Corollary 2.5]. Also, if $A(t) \equiv I_{n}$, this result was obtained in [6] and [11] ( $c f$. [24] in the case of the maximum norm in $\left.\mathbb{C}^{n}\right)$. 
Corollary 2.4. Let $A:[0, \infty) \rightarrow L\left(\mathbb{C}^{n}, \mathbb{C}^{n}\right)$ be a measurable mapping which satisfies the assumptions of Definition 1.5 and the condition (2.6). Assume $f$ : $B^{n} \rightarrow \mathbb{C}^{n}$ has generalized parametric representation with respect to $A$. Then there exists a univalent subordination chain $f(z, t)$ such that $D f(0, t)=e^{\int_{0}^{t} A(\tau) d \tau}$, $t \geq 0,\left\{e^{-\int_{0}^{t} A(\tau) d \tau} f(\cdot, t)\right\}_{t \geq 0}$ is a normal family on $B^{n}$ and $f=f(\cdot, 0)$.

Proof. It suffices to consider the univalent subordination chain $f(z, t)$ given by(2.7). Since $D f(0, t)=e^{\int_{0}^{t} A(\tau) d \tau},\left\{e^{-\int_{0}^{t} A(\tau) d \tau} f(\cdot, t)\right\}_{t \geq 0}$ is a normal family on $B^{n}$ and $f=f(\cdot, 0)$ by Theorem 2.3 , the conclusion follows.

Now we prove that if $A:[0, \infty) \rightarrow L\left(\mathbb{C}^{n}, \mathbb{C}^{n}\right)$ is a measurable mapping which satisfies the conditions in Definition 1.5 and the assumption (2.6), then the solutions of the generalized Loewner differential equation (2.12) that satisfy a normal family condition provide univalent subordination chains which have generalized parametric representation with respect to $A$. This result is a generalization of [8, Theorem 2.6] (see also [19, Theorems 2.2 and 2.3] and [25, Theorem 6]). In the case of one complex variable, compare with [21].

Theorem 2.5. Let $A:[0, \infty) \rightarrow L\left(\mathbb{C}^{n}, \mathbb{C}^{n}\right)$ be a measurable mapping which satisfies the assumptions of Definition 1.5 and the condition (2.6). Also let $f=$ $f(z, t): B^{n} \times[0, \infty) \rightarrow \mathbb{C}^{n}$ be a mapping such that $f(\cdot, t) \in H\left(B^{n}\right), f(0, t)=0$, $D f(0, t)=e^{\int_{0}^{t} A(\tau) d \tau}$ for $t \geq 0$, and $f(z, \cdot)$ is locally absolutely continuous on $[0, \infty)$ locally uniformly with respect to $z \in B^{n}$. Let $h=h(z, t): B^{n} \times[0, \infty) \rightarrow$ $\mathbb{C}^{n}$ satisfy the assumptions of Theorem 2.1. Assume $f(z, t)$ satisfies the Loewner differential equation

$$
\frac{\partial f}{\partial t}(z, t)=D f(z, t) h(z, t) \text { a.e. } t \geq 0, \forall z \in B^{n} .
$$

Also assume that $\left\{e^{-\int_{0}^{t} A(\tau) d \tau} f(\cdot, t)\right\}_{t \geq 0}$ is a normal family on $B^{n}$. Then $f(z, t)$ is a univalent subordination chain such that $f(z, s)=f(v(z, s, t), t)$ and

$$
f(z, s)=\lim _{t \rightarrow \infty} e^{\int_{0}^{t} A(\tau) d \tau} v(z, s, t)
$$

locally uniformly on $B^{n}$ for $s \geq 0$, where $v=v(z, s, t)$ is the unique locally Lipschitz continuous solution on $[s, \infty)$ of the initial value problem

$$
\frac{\partial v}{\partial t}=-h(v, t) \text { a.e. } t \geq s, v(z, s, s)=z
$$

for all $s \geq 0$ and $z \in B^{n}$. Moreover, $f(\cdot, 0)$ has generalized parametric representation with respect to $A$.

Proof. Let $v=v(z, s, t)$ be the unique Lipschitz continuous solution of the initial value problem

$$
\frac{\partial v}{\partial t}=-h(v, t) \text { a.e. } t \geq s, v(z, s, s)=z
$$


for all $z \in B^{n}$ and $s \geq 0$. Also let $f(z, s, t)=f(v(z, s, t), t)$ for $z \in B^{n}$ and $t \geq s \geq 0$. We may prove that $f(z, s, t)=f(z, s, s)$, i.e. $f(v(z, s, t), t)=f(z, s)$ for $z \in B^{n}$ and $t \geq s \geq 0$, by the same argument as in the proof of [8, Theorem 2.6] (see also the proof of [19, Theorem 2.2]).

We next prove that $f(\cdot, t)$ is biholomorphic on $B^{n}$ and $f(\cdot, 0)$ has generalized parametric representation with respect to $A$. To this end, we prove that there exists a sequence $\left\{t_{m}\right\}_{m \in \mathbb{N}}$ such that $0<t_{m} \rightarrow \infty$ and

$$
\lim _{m \rightarrow \infty} e^{\int_{0}^{t_{m}} A(\tau) d \tau} v\left(z, s, t_{m}\right)=f(z, s)
$$

locally uniformly on $B^{n}$. Indeed, since $\left\{e^{-\int_{0}^{t} A(\tau) d \tau} f(\cdot, t)\right\}_{t \geq 0}$ is a normal family on $B^{n}$, we deduce that for each $r \in(0,1)$ there exists a constant $K(r)>0$ such that

$$
\left\|e^{-\int_{0}^{t} A(\tau) d \tau} f(z, t)\right\| \leq K(r),\|z\| \leq r, t \geq 0 .
$$

Hence

$$
\left\|e^{-\int_{0}^{t} A(\tau) d \tau} f(z, t)-z\right\| \leq(K(r)+r) \frac{\|z\|^{2}}{r^{2}},\|z\| \leq r, t \geq 0 .
$$

Replacing $z$ by $v(z, s, t)$ in the above inequality and using the relation (2.2) and the fact that $f(z, s)=f(v(z, s, t), t)$, we obtain that

$$
\begin{aligned}
\left\|f(z, s)-e^{\int_{0}^{t} A(\tau) d \tau} v(z, s, t)\right\| & =\left\|e^{\int_{0}^{t} A(\tau) d \tau}\left[e^{-\int_{0}^{t} A(\tau) d \tau} f(v(z, s, t), t)-v(z, s, t)\right]\right\| \\
& \leq\left\|e^{\int_{0}^{t} A(\tau) d \tau}\right\| \frac{K(r)+r}{r^{2}}\|v(z, s, t)\|^{2} \\
& \leq \frac{K(r)+r}{(1-r)^{4}}\left\|e^{\int_{0}^{t}\left[A(\tau)-2 m(A(\tau)) I_{n}\right] d \tau}\right\| e^{2 \int_{0}^{s} m(A(\tau)) d \tau} .
\end{aligned}
$$

Since $\int_{0}^{\infty}\left\|e^{\int_{0}^{t}\left[A(\tau)-2 m(A(\tau)) I_{n}\right] d \tau}\right\| d t<\infty$, there exists a sequence $\left\{t_{m}\right\}_{m \in \mathbb{N}}$ such that $0<t_{m} \rightarrow \infty$ and $\left\|e^{\int_{0}^{t_{m}}\left[A(\tau)-2 m(A(\tau)) I_{n}\right] d \tau}\right\| \rightarrow 0$ as $m \rightarrow \infty$. Hence the relation (2.14) holds. On the other hand, since the $\operatorname{limit}_{\lim _{t \rightarrow \infty}} e^{\int_{0}^{t} A(\tau) d \tau} v(z, s, t)=$ $g(z, s)$ exists locally uniformly on $B^{n}$ by Theorem 2.3 and $g(z, s)$ is a univalent subordination chain such that $g(\cdot, 0)$ has generalized parametric representation with respect to $A$, we deduce from the above relations that $f(z, t)=g(z, t)$, and thus $f(z, t)$ is a univalent subordination chain such that $f(\cdot, 0)$ has generalized parametric representation with respect to $A$. This completes the proof.

We next prove the converse of Theorem 2.3 (cf. [8, Theorem 2.8]; compare with [6, Theorem 1.10] and [11, Theorem 2.2]).

Theorem 2.6. Let $A:[0, \infty) \rightarrow L\left(\mathbb{C}^{n}, \mathbb{C}^{n}\right)$ be a measurable mapping which satisfies the assumptions of Definition 1.5 and the relation (2.6). Also let $f(z, t)$ be a univalent subordination chain such that $D f(0, t)=e^{\int_{0}^{t} A(\tau) d \tau}$ for $t \geq 0$, 
and let $v=v(z, s, t)$ be the transition mapping associated with $f(z, t)$. Assume $\left\{e^{-\int_{0}^{t} A(\tau) d \tau} f(\cdot, t)\right\}_{t \geq 0}$ is a normal family on $B^{n}$. Then the limit

$$
\lim _{t \rightarrow \infty} e^{\int_{0}^{t} A(\tau) d \tau} v(z, s, t)=f(z, s)
$$

exists locally uniformly on $B^{n}$ for $s \geq 0$. Moreover, $f(\cdot, 0)$ has generalized parametric representation with respect to $A$.

Proof. Fix $r \in(0,1)$ and $t>s \geq 0$. Since $\|A(\cdot)\|$ is uniformly bounded on $[0, \infty)$, it follows that there exists $b>0$ such that $\|A(t)\| \leq b$ for $t \geq 0$. Let $g_{s, t}(z)=z-v(z, s, t)$ for $z \in B^{n}$. Then $D g_{s, t}(0)=I_{n}-e^{-\int_{s}^{t} A(\tau) d \tau}$ and $g_{s, t} \in \mathcal{N}$. Also $m\left(I_{n}-e^{-\int_{s}^{t} A(\tau) d \tau}\right)>0$. Indeed, fix $u \in \mathbb{C}^{n}$ such that $\|u\|=1$ and let $q(\tau)=\left\|e^{-\int_{s}^{\tau} A(\xi) d \xi}(u)\right\|^{2}$ for $\tau \geq s$. Then $q$ is locally Lipschitz continuous on $[s, \infty)$, since $\|A(t)\| \leq b$ for $t \geq 0$. Hence there exists $(d q / d \tau)(\tau)$ for almost all $\tau \geq s$. Since $m(A(t))>0$ for $t \geq 0$ and the mapping $A$ satisfies the condition (1.1), we deduce that

$$
A(\tau) \circ e^{-\int_{s}^{\tau} A(\xi) d \xi}=e^{-\int_{s}^{\tau} A(\xi) d \xi} \circ A(\tau), \text { a.e. } \tau \geq s,
$$

and hence

$$
\begin{aligned}
q^{\prime}(\tau) & =2 \Re\left\langle\frac{d}{d \tau} e^{-\int_{s}^{\tau} A(\xi) d \xi}(u), e^{-\int_{s}^{\tau} A(\xi) d \xi}(u)\right\rangle \\
& =-2 \Re\left\langle A(\tau) e^{-\int_{s}^{\tau} A(\xi) d \xi}(u), e^{-\int_{s}^{\tau} A(\xi) d \xi}(u)\right\rangle<0, \text { a.e. } \tau \geq s .
\end{aligned}
$$

Hence $q(\tau)<1$ for $\tau>$ s, i.e. $\left\|e^{-\int_{s}^{\tau} A(\xi) d \xi}\right\|<1$ for $\tau>s$, and thus

$$
m\left(I_{n}-e^{-\int_{s}^{t} A(\tau) d \tau}\right)=\min _{\|z\|=1}\left\{1-\Re\left\langle e^{-\int_{s}^{t} A(\tau) d \tau}(z), z\right\rangle\right\} \geq 1-\left\|e^{-\int_{s}^{t} A(\tau) d \tau}\right\|>0,
$$

as desired.

Next, in view of Lemma 1.2, we deduce that

$$
\left\|g_{s, t}(z)\right\| \leq \frac{4 r}{(1-r)^{2}}\left|V\left(I_{n}-e^{-\int_{s}^{t} A(\tau) d \tau}\right)\right| \leq \frac{4 r}{(1-r)^{2}}\left\|I_{n}-e^{-\int_{s}^{t} A(\tau) d \tau}\right\|
$$

for $\|z\| \leq r$. Now, since $\|A(t)\| \leq b$ for $t \geq 0$, we obtain that

$$
\left\|I_{n}-e^{-\int_{s}^{t} A(\tau) d \tau}\right\| \leq \sup _{\tau \in[s, t]}\left\{\|A(\tau)\| \cdot\left\|e^{-\int_{s}^{\tau} A(\xi) d \xi}\right\|\right\}(t-s) \leq b(t-s),
$$

and hence

$$
\left\|g_{s, t}(z)\right\| \leq \frac{4 r b}{(1-r)^{2}}(t-s),\|z\| \leq r, t \geq s \geq 0
$$


Equivalently

$$
\|z-v(z, s, t)\| \leq \frac{4 r b}{(1-r)^{2}}(t-s),\|z\| \leq r, t \geq s \geq 0 .
$$

On the other hand, using the Cauchy integral formula and the fact that $v(\cdot, s, t)$ is a Schwarz mapping, we deduce that for each $r \in(0,1)$ there exists $L=L(r)>0$ such that

$$
\|v(z, s, t)-v(w, s, t)\| \leq L(r)\|z-w\|, z, w \in \bar{B}_{r}^{n}, t \geq s \geq 0 .
$$

Replacing $w$ by $v\left(z, s_{1}, s_{2}\right)$ in the above relation and using the semigroup property of the transition mapping, we deduce in view of (2.15) that

$$
\begin{aligned}
& \left\|v\left(z, s_{2}, t\right)-v\left(z, s_{1}, t\right)\right\|=\left\|v\left(z, s_{2}, t\right)-v\left(v\left(z, s_{1}, s_{2}\right), s_{2}, t\right)\right\| \\
& \leq L(r) \frac{4 r b}{(1-r)^{2}}\left(s_{2}-s_{1}\right)=L^{*}(r, A)\left(s_{2}-s_{1}\right), 0 \leq s_{1} \leq s_{2} \leq t,\|z\| \leq r .
\end{aligned}
$$

Next, we may prove that $f(z, \cdot)$ is locally Lipschitz continuous on $[0, \infty)$ locally uniformly with respect to $z \in B^{n}$ as in the proof of [8, Theorem 2.8]. Finally, using arguments similar to those in the proof of [6, Theorem 1.10], we deduce that there exists a mapping $h=h(z, t)$ such that $h(\cdot, t) \in \mathcal{N}, D h(0, t)=A(t)$ for $t \geq 0$, $h(z, \cdot)$ is measurable on $[0, \infty)$ for $z \in B^{n}$, and

$$
\frac{\partial f}{\partial t}(z, t)=D f(z, t) h(z, t) \text { a.e. } t \geq 0, \forall z \in B^{n} .
$$

Finally, in view of Theorem 2.5, the conclusion follows.

Combining Theorem 2.6 and Corollary 2.4, we deduce the following result, which yields the equivalence between the notions of generalized parametric representation with respect to a measurable linear operator and non-normalized univalent subordination chains ( $c f$. [8, Corollary 2.9]; compare with [11, Corollary 2.5] and [24]).

Corollary 2.7. Let $A:[0, \infty) \rightarrow L\left(\mathbb{C}^{n}, \mathbb{C}^{n}\right)$ be a measurable mapping which satisfies the assumptions of Definition 1.5 and the condition (2.6). Also let $f \in$ $S\left(B^{n}\right)$. Then $f$ has generalized parametric representation with respect to $A$ if and only if there exists a univalent subordination chain $f(z, t)$ such that $D f(0, t)=$ $e^{\int_{0}^{t} A(\tau) d \tau},\left\{e^{-\int_{0}^{t} A(\tau) d \tau} f(\cdot, t)\right\}_{t \geq 0}$ is a normal family on $B^{n}$ and $f=f(\cdot, 0)$.

In view of (2.2) and (2.3), we deduce the following growth result for mappings in $\tilde{S}_{A}^{0}\left(B^{n}\right)$, where $A$ is a measurable diagonal matrix with its eigenvalues $a_{1}, \ldots, a_{n}$, such that $\Re a_{1}(t)=\cdots=\Re a_{n}(t)$ for $t \geq 0$ (if $A=I_{n}$, see [23] and [6]). 
Remark 2.8. Let $a_{j}:[0, \infty) \rightarrow \mathbb{C}$ be uniformly bounded measurable functions for $j=1, \ldots, n$, such that $\Re a_{1}(t)=\cdots=\Re a_{n}(t) \geq \delta$ for some $\delta>0$. If $A:[0, \infty) \rightarrow L\left(\mathbb{C}^{n}, \mathbb{C}^{n}\right)$ is a diagonal matrix with eigenvalues $a_{1}, \ldots, a_{n}$ and if $f \in \tilde{S}_{A}^{0}\left(B^{n}\right)$, then

$$
\frac{\|z\|}{(1+\|z\|)^{2}} \leq\|f(z)\| \leq \frac{\|z\|}{(1-\|z\|)^{2}}, z \in B^{n} .
$$

Proof. It is clear that the matrix-valued mapping $A$ satisfies the assumptions of Definition 1.5. Since $f$ has generalized parametric representation with respect to $A$, there exists a mapping $h=h(z, t): B^{n} \times[0, \infty) \rightarrow \mathbb{C}^{n}$ such that $h(\cdot, t) \in \mathcal{N}$, $D h(0, t)=A(t)$ for $t \geq 0, h(z, \cdot)$ is measurable on $[0, \infty)$ for $z \in B^{n}$, and $f(z)=\lim _{t \rightarrow \infty} e^{\int_{0}^{t} A(\tau) d \tau} v(z, t)$ locally uniformly on $B^{n}$, where $v=v(z, t)$ is the unique locally Lipschitz continuous solution on $[0, \infty)$ of the initial value problem

$$
\frac{\partial v}{\partial t}=-h(v, t) \text { a.e. } t \geq 0, v(z, 0)=z
$$

On the other hand, since $A(t)$ is diagonal and its eigenvalues satisfy the equality $\Re a_{1}(t)=\cdots=\Re a_{n}(t)$, it follows that

$$
\|f(z)\|=\lim _{t \rightarrow \infty}\left\|e^{\int_{0}^{t} A(\tau) d \tau} v(z, t)\right\|=\lim _{t \rightarrow \infty} e^{\int_{0}^{t} \Re a_{1}(\tau) d \tau}\|v(z, t)\|, z \in B^{n} .
$$

Finally, taking into account the relations (2.2) and (2.3), the conclusion follows.

\section{Generalized spirallike mappings and non-normalized univalent subordination chains}

In this section we apply the above results to introduce a generalization of the usual notion of spirallikeness on $B^{n}$, and to investigate the connection between this notion and univalent subordination chains.

Definition 3.1. Let $A:[0, \infty) \rightarrow L\left(\mathbb{C}^{n}, \mathbb{C}^{n}\right)$ be a locally Lebesgue integrable mapping such that $m(A(t))>0$ for $t \geq 0$. Also let $\Omega$ be a domain in $\mathbb{C}^{n}$ which contains the origin. We say that $\Omega$ is generalized spirallike with respect to $A$ if $e^{-\int_{s}^{t} A(\tau) d \tau}(w) \in \Omega$, for all $w \in \Omega$ and $t \geq s \geq 0$.

A mapping $f \in S\left(B^{n}\right)$ is called generalized spirallike with respect to $A$ if $f\left(B^{n}\right)$ is a generalized spirallike domain with respect to $A$.

Remark 3.2. Clearly if $A(t)$ is constant, we obtain the usual notion of spirallikeness with respect to a given constant operator $A$. In particular, if $A(t) \equiv I_{n}$ in Definition 3.1, then $\Omega$ is a starlike domain.

We next obtain the following characterization of generalized spirallikeness with respect to a given measurable operator in terms of univalent subordination chains. 
Theorem 3.3. Let $A:[0, \infty) \rightarrow L\left(\mathbb{C}^{n}, \mathbb{C}^{n}\right)$ be a locally Lebesgue integrable mapping such that $m(A(t))>0$ for $t \geq 0$ and the condition (1.1) holds. Also let $f \in H\left(B^{n}\right)$ be a normalized mapping. Then $f$ is generalized spirallike with respect to $A$ if and only if $f(z, t)=e^{\int_{0}^{t} A(\tau) d \tau} f(z)$ is a univalent subordination chain.

Proof. It suffices to apply Definition 3.1 and the notion of a univalent subordination chain.

In view of Theorem 3.3, we deduce the following consequence, which yields that generalized spirallike mappings with respect to measurable linear operators, that satisfy the assumptions of Definition 1.5 and the condition (2.6), have generalized parametric representation. If $A(t)$ is constant, we obtain [8, Corollary 2.12]. Recall that in dimension $n \geq 2$, there exist spirallike mappings with respect to a given linear operator which do not belong to the class $S^{0}\left(B^{n}\right)$ (see [7]).

Corollary 3.4. Let $A:[0, \infty) \rightarrow L\left(\mathbb{C}^{n}, \mathbb{C}^{n}\right)$ be a measurable mapping which satisfies the assumptions of Definition 1.5 and the relation (2.6). Also let $f: B^{n} \rightarrow$ $\mathbb{C}^{n}$ be a generalized spirallike mapping with respect to $A$. Then $f$ has generalized parametric representation with respect to $A$.

Proof. Since $\|A(\cdot)\|$ is uniformly bounded on $[0, \infty)$ and the mapping $A$ is measurable, it follows that $A$ is locally Lebesgue integrable on $[0, \infty)$. Taking into account Theorem 3.3, we deduce that $f(z, t)=e^{\int_{0}^{t} A(\tau) d \tau} f(z)$ is a univalent subordination chain, and since $f \in S\left(B^{n}\right),\left\{e^{-\int_{0}^{t} A(\tau) d \tau} f(\cdot, t)\right\}_{t \geq 0}$ is a normal family on $B^{n}$. Consequently, $f$ has generalized parametric representation with respect to $A$ by Corollary 2.7 .

We next obtain the following analytical characterization of generalized spirallikeness with respect to a given measurable operator. If $A(t)$ is constant, we obtain the usual characterization of spirallikeness due to Suffridge [30] (see also [12]).

Theorem 3.5. Let $A:[0, \infty) \rightarrow L\left(\mathbb{C}^{n}, \mathbb{C}^{n}\right)$ be a measurable mapping which satisfies the assumptions of Definition 1.5. Also let $f \in \mathcal{L} S_{n}$. Then $f$ is generalized spirallike with respect to $A$ if and only if

$$
\Re\left\langle[D f(z)]^{-1} A(t) f(z), z\right\rangle>0, \text { a.e. } t \geq 0, \forall z \in B^{n} \backslash\{0\} .
$$

Proof. First assume that $f$ is generalized spirallike with respect to $A$. Since $\|A(t)\|$ is uniformly bounded, it follows that the mapping $q_{t}(\tau)=\exp \left(-\int_{t}^{t+\tau} A(\xi) d \xi\right)$ is locally Lipschitz continuous on $[0, \infty)$ for each $t \geq 0$, and

$$
\lim _{\tau \rightarrow 0+} \frac{I_{n}-e^{-\int_{t}^{t+\tau} A(\xi) d \xi}}{\tau}=A(t), \text { a.e. } t \geq 0 .
$$

Fix $t \geq 0$ such that the above relation holds, and let $F(z, \tau)=e^{-\int_{t}^{t+\tau} A(\xi) d \xi} f(z)$ for $z \in B^{n}$ and $\tau \geq 0$. Since $f$ is generalized spirallike with respect to $A$, 
$F\left(B^{n}, \tau\right) \subseteq f\left(B^{n}\right)$ for $\tau \geq 0$. Also $F(z, 0)=f(z), F(0, \tau)=0$ and

$$
\lim _{\tau \rightarrow 0+} \frac{F(z, 0)-F(z, \tau)}{\tau}=A(t) f(z)
$$

locally uniformly on $B^{n}$. Taking into account [29, Lemma 2], we deduce that

$$
\Re\left\langle[D f(z)]^{-1} A(t) f(z), z\right\rangle \geq 0, z \in B^{n} .
$$

Next, fix $w \in \mathbb{C}^{n},\|w\|=1$, and let $r: U \rightarrow \mathbb{C}$ be given by

$$
r(\zeta)= \begin{cases}\frac{1}{\zeta}\left\langle[D f(\zeta w)]^{-1} A(t) f(\zeta w), w\right\rangle, & 0<|\zeta|<1 \\ \langle A(t) w, w\rangle, & \zeta=0 .\end{cases}
$$

Then $r$ is a holomorphic function on the unit disc, $\Re r(\zeta) \geq 0$ for $0<|\zeta|<1$, and since $m(A(t))>0$ it follows that $\operatorname{Rr}(0)>0$. Taking into account the minimum principle for harmonic functions, we deduce that $\Re r(\zeta)>0$ for $|\zeta|<1$. Therefore

$$
\Re\left\langle[D f(z)]^{-1} A(t) f(z), z\right\rangle>0, z \in B^{n} \backslash\{0\} .
$$

Conversely, assume that the relation (3.1) holds. Let $E \subset[0, \infty)$ be a null set such that the relation (3.1) holds for all $t \in[0, \infty) \backslash E$ and for all $z \in B^{n}$. Also let $h=h(z, t): B^{n} \times[0, \infty) \rightarrow \mathbb{C}^{n}$ be given by

$$
h(z, t)= \begin{cases}{[D f(z)]^{-1} A(t) f(z),} & z \in B^{n}, t \in[0, \infty) \backslash E \\ A(t)(z), & z \in B^{n}, t \in E .\end{cases}
$$

Then $h(z, t)$ satisfies the assumptions of Theorem 2.1. Fix $s \geq 0$ and $z \in B^{n}$, and let $v=v(z, s, t)$ be the unique solution of the initial value problem

$$
\frac{\partial v}{\partial t}=-h(v, t), \text { a.e. } t \geq s, v(z, s, s)=z
$$

Since $\int_{0}^{\infty} m(A(t)) d t=\infty$, it follows in view of (2.2) that $\|v(z, s, t)\| \rightarrow 0$ as $t \rightarrow \infty$. On the other hand, $f$ is biholomorphic on $B^{n}$, since $f$ is spirallike with respect to each operator $A(t)$ for almost all $t \in[0, \infty)$. Setting $u(z, s, t)=$ $f^{-1}\left(e^{-\int_{s}^{t} A(\tau) d \tau} f(z)\right)$ for $t$ sufficiently close to $s$, we obtain that $u(z, s, t)$ is a solution of (3.2). The uniqueness of solutions to (3.2) and the semigroup property yield the equality $u(z, s, t)=v(z, s, t)$ for all $t \geq s$. Thus $u(\cdot, s, t)$ is a Schwarz mapping, in view of Theorem 2.1. Therefore $e^{-\int_{s}^{t} A(\tau) d \tau} f\left(B^{n}\right) \subseteq f\left(B^{n}\right)$ for $t \geq s$, as desired. This completes the proof. 


\section{Remark 3.6.}

(i) Let $A:[0, \infty) \rightarrow L\left(\mathbb{C}^{n}, \mathbb{C}^{n}\right)$ be a measurable mapping which satisfies the assumptions of Definition 1.5. In view of Theorem 3.5, we deduce that a mapping $f \in \mathcal{L} S_{n}$ is generalized spirallike with respect to $A$ if and only if $f$ is spirallike with respect to each operator $A(t)$ for almost all $t \geq 0$.

(ii) Let $f \in S\left(B^{n}\right)$ be a generalized spirallike mapping with respect to $A$. Also let $E \subset[0, \infty)$ be such that $\lambda(E)=0$ and $f$ is spirallike with respect to each operator $A(t)$ for $t \in[0, \infty) \backslash E$. Here $\lambda$ is the usual Lebesgue measure on $\mathbb{R}$. If there exists $t_{0} \in[0, \infty) \backslash E$ such that $A\left(t_{0}\right)+\left[A\left(t_{0}\right)\right]^{*}=2 I_{n}$, then $f \in S^{0}\left(B^{n}\right)$ by [8, Theorem 3.14]. Here $\left[A\left(t_{0}\right)\right]^{*}$ is the adjoint operator of $A\left(t_{0}\right)$.

If $A=\exp (-i \alpha) I_{n}$ in Theorem 3.5, where $\alpha:[0, \infty) \rightarrow[-\delta, \delta]$ is a measurable function and $\delta \in(0, \pi / 2)$, then we obtain the following result ( $c f$. [14]):

Corollary 3.7. Let $\delta \in(0, \pi / 2)$ and $\alpha:[0, \infty) \rightarrow[-\delta, \delta]$ be a measurable function. Also let $f \in \mathcal{L} S_{n}$ and $A(t)=e^{-i \alpha(t)} I_{n}$ for $t \geq 0$. Then $f$ is generalized spirallike with respect to $A$ if and only if

$$
\mathfrak{R}\left[e^{-i \alpha(t)}\left\langle[D f(z)]^{-1} f(z), z\right\rangle\right]>0, \text { a.e. } t \geq 0, \forall z \in B^{n} \backslash\{0\} .
$$

Proof. Elementary computations yield that $\|A(t)\|=1, m(A(t))=\cos \alpha(t)$ for $t \geq 0$, and $\int_{0}^{\infty} m(A(t)) d t=\infty$. Therefore the mapping $A$ satisfies the assumptions of Definition 1.5. In view of Theorem 3.5, we deduce that $f$ is generalized spirallike with respect to $A$ if and only if the relation (3.3) holds, as desired.

Remark 3.8. Let $f$ be a generalized spirallike mapping with respect to $A$, where $A(t)=e^{-i \alpha(t)} I_{n}$ for $t \geq 0$, and $\alpha$ satisfies the assumptions of Corollary 3.7. Then $f$ is spirallike of type $\alpha(t)$ for almost all $t \geq 0$, and hence

$$
\frac{\|z\|}{(1+\|z\|)^{2}} \leq\|f(z)\| \leq \frac{\|z\|}{(1-\|z\|)^{2}}, z \in B^{n},
$$

by Remark 2.8. However, if $f$ is a spirallike mapping with respect to a given measurable linear operator $A$, then $f$ does not necessarily satisfy the above growth result [15].

\section{Examples of generalized spirallike mappings with respect to diagonal operators}

We next consider certain examples of generalized spirallike mappings with respect to measurable diagonal matrices. The proof of the first example is a direct application of the notion of generalized spirallikeness. 
Example 4.1. Let $\alpha_{j}:[0, \infty) \rightarrow \mathbb{C}$ be a locally Lebesgue integrable function on $[0, \infty)$ with $\mathfrak{i} \alpha_{j}(t)>0$ for $t \geq 0$. If $f_{j}$ is a generalized spirallike function with respect to $\alpha_{j}$ for $j=1, \ldots, n$, then the mapping $f: B^{n} \rightarrow \mathbb{C}^{n}$ given by $f(z)=\left(f_{1}\left(z_{1}\right), \ldots, f_{n}\left(z_{n}\right)\right)$ is generalized spirallike with respect to $A$, where $A(t)=\operatorname{diag}\left(\alpha_{1}(t), \ldots, \alpha_{n}(t)\right)$ for $t \geq 0$.

Example 4.2. Let $\delta \in(0, \pi / 2)$ and $\alpha:[0, \infty) \rightarrow[-\delta, \delta]$ be a measurable function. Also let $a(t)=e^{-i \alpha(t)}$ for $t \geq 0$. If $\lambda \in \mathbb{C}$ is such that $|\lambda| \leq \cos \delta$, then the function $f: U \rightarrow \mathbb{C}$ given by $f\left(z_{1}\right)=z_{1} /\left(1-\lambda z_{1}\right)$ is generalized spirallike with respect to the function $a$.

Proof. It suffices to apply Theorem 3.5 to show that $f\left(z_{1}\right)=z_{1} /\left(1-\lambda z_{1}\right)$ is generalized spirallike with respect to the function $a$.

Example 4.3. In view of Examples 4.1 and 4.2, we deduce that if $\delta_{j} \in(0, \pi / 2)$, $\alpha_{j}:[0, \infty) \rightarrow\left[-\delta_{j}, \delta_{j}\right]$ is a measurable function and $\left|\lambda_{j}\right| \leq \cos \delta_{j}$ for $j=$ $1, \ldots, n$, then the mapping $F: B^{n} \rightarrow \mathbb{C}^{n}$,

$$
F(z)=\left(\frac{z_{1}}{1-\lambda_{1} z_{1}}, \ldots, \frac{z_{n}}{1-\lambda_{n} z_{n}}\right), z=\left(z_{1}, \ldots, z_{n}\right) \in B^{n},
$$

is generalized spirallike with respect to $A$, where $A(t)=\operatorname{diag}\left(e^{-i \alpha_{1}(t)}, \ldots, e^{-i \alpha_{n}(t)}\right)$ for $t \geq 0$.

Remark 4.4. Under the same assumptions as in Example 4.3, the mapping $G$ : $B^{n} \rightarrow \mathbb{C}^{n}$ given by $G(z)=\left(z_{1} e^{\lambda_{1} z_{1}}, \ldots, z_{n} e^{\lambda_{n} z_{n}}\right)$ is generalized spirallike with respect to the operator $A$, where $A(t)=\operatorname{diag}\left(\alpha_{1}(t), \ldots, \alpha_{n}(t)\right)$ for $t \geq 0$.

The next example of a generalized spirallike mapping with respect to a measurable diagonal matrix is provided by the Roper-Suffridge extension operator. This operator was introduced by Roper and Suffridge [28], as follows: let $\Phi_{n}: \mathcal{L} S \rightarrow$ $\mathcal{L} S_{n}$ be given by

$$
\Phi_{n}(f)(z)=\left(f\left(z_{1}\right), \tilde{z} \sqrt{f^{\prime}\left(z_{1}\right)}\right), z=\left(z_{1}, \tilde{z}\right) \in B^{n} .
$$

Here we choose the principal branch of the power function. One of the main properties of the operator $\Phi_{n}$ is that it preserves convexity from dimension one to the $n$-dimensional case, i.e. if $f$ is convex on $U$ then $\Phi_{n}(f)$ is also convex on $B^{n}$ (see [28]). We have

Example 4.5. Let $b, \delta>0$ and $\alpha:[0, \infty) \rightarrow \mathbb{C}$ be a measurable function such that $\Re \alpha(t) \geq \delta$ and $|\alpha(t)| \leq b$ for $t \geq 0$. Also let $f$ be a generalized spirallike function with respect to $\alpha$ and let $A:[0, \infty) \rightarrow L\left(\mathbb{C}^{n}, \mathbb{C}^{n}\right)$ be given by $A(t)=\alpha(t) I_{n}$ for $t \geq 0$. Then $\Phi_{n}(f)$ is generalized spirallike with respect to $A$.

Proof. It is obvious that the mapping $A$ satisfies the assumptions of Definition 1.5. Since $f$ is generalized spirallike with respect to $\alpha$, it follows that $f$ is spirallike with respect to $\alpha(t)$ for almost all $t \geq 0$, by Remark 3.6 (i). Then $\Phi_{n}(f)$ is spirallike with respect to $A(t)$ for almost all $t \geq 0$ by [10, Corollary 2.3]. Hence $\Phi_{n}$ is generalized spirallike with respect to $A$, as desired. 
We next consider conditions under which the mapping $F: B^{n} \rightarrow \mathbb{C}^{n}$ given by $F(z)=P(z)(z)$ is generalized spirallike with respect to a measurable diagonal operator $A$, where $P: B^{n} \rightarrow \mathbb{C}$ is a holomorphic function with $P(0)=1$. We have (cf. [20])

\section{Example 4.6.}

(i) Let $\delta \in(0, \pi / 2)$ and $\alpha:[0, \infty) \rightarrow[-\delta, \delta]$ be a measurable function. Then $F(z)=P(z)(z)$ is generalized spirallike with respect to $A:[0, \infty) \rightarrow$ $L\left(\mathbb{C}^{n}, \mathbb{C}^{n}\right)$ given by $A(t)=e^{-i \alpha(t)} I_{n}$ if and only if

$$
\Re\left[e^{i \alpha(t)}\left\{1+\frac{D P(z)(z)}{P(z)}\right\}\right]>0 \text {, a.e. } t \geq 0, \forall z \in B^{n} .
$$

(ii) In particular, if $f_{j}$ is generalized spirallike on $U$ with respect to the function $a:[0, \infty) \rightarrow \mathbb{C}$ given by $a(t)=e^{-i \alpha(t)}$ for $j=1, \ldots, n$, and if $\lambda_{j} \geq 0$, $\sum_{j=1}^{n} \lambda_{j}=1$, then the mapping $F: B^{n} \rightarrow \mathbb{C}^{n}$, given by

$$
F(z)=z \prod_{j=1}^{n}\left(\frac{f_{j}\left(z_{j}\right)}{z_{j}}\right)^{\lambda_{j}}, z=\left(z_{1}, \ldots, z_{n}\right) \in B^{n},
$$

is generalized spirallike with respect to $A$ given by $A(t)=e^{-i \alpha(t)} I_{n}$.

Proof. Let $L(z)=D P(z) / P(z)$. After elementary computations, we obtain that

$$
[D F(z)]^{-1}=\frac{1}{P(z)}\left[I_{n}-\frac{z L(z)(\cdot)}{1+L(z)(z)}\right], z \in B^{n} .
$$

Then

$$
\Re\left\langle[D F(z)]^{-1} A(t) F(z), z\right\rangle=\Re\left[\frac{e^{-i \alpha(t)}\|z\|^{2}}{1+L(z)(z)}\right], z \in B^{n}, t \geq 0 .
$$

In view of Theorem 3.5, we deduce that $F$ is generalized spirallike with respect to $A$ if and only if $\Re\left[e^{i \alpha(t)}(1+L(z)(z))\right]>0$ for almost all $t \geq 0$ and for all $z \in B^{n}$, i.e. if and only if the relation (4.1) holds.

To deduce (ii), it suffices to apply (4.1).

The following result provides other examples of generalized spirallike mappings on $B^{n}$ with respect to a given measurable diagonal operator.

Theorem 4.7. Let $\alpha \in[0,1], b, \eta>0$ and $\lambda:[0, \infty) \rightarrow \mathbb{C}$ be a measurable function such that $|\lambda(t)| \leq b$ and $\mathfrak{R} \lambda(t) \geq \eta$ for $t \geq 0$. Also let $f: U \rightarrow \mathbb{C}$ be $a$ generalized spirallike function with respect to $\lambda$ and let $F_{\alpha}: B^{n} \rightarrow \mathbb{C}^{n}$ be given by

$$
F_{\alpha}(z)=\left(f\left(z_{1}\right), \tilde{z}\left(\frac{f\left(z_{1}\right)}{z_{1}}\right)^{\alpha}\right), z=\left(z_{1}, \tilde{z}\right) \in B^{n} .
$$

Then $F_{\alpha}$ is generalized spirallike with respect to $A$, where $A(t)=\lambda(t) I_{n}$. 
Proof. It suffices to assume $n=2$. By direct computations we have

$$
\left[D F_{\alpha}(z)\right]^{-1} A(t) F_{\alpha}(z)=\left(\frac{\lambda(t) f\left(z_{1}\right)}{f^{\prime}\left(z_{1}\right)}, \lambda(t) z_{2}(1-\alpha)+\lambda(t) \alpha z_{2} \frac{f\left(z_{1}\right)}{z_{1} f^{\prime}\left(z_{1}\right)}\right) .
$$

Hence

$$
\begin{aligned}
& \Re\left\langle\left[D F_{\alpha}(z)\right]^{-1} A(t) F_{\alpha}(z), z\right\rangle \\
& =\left(\left|z_{1}\right|^{2}+\alpha\left|z_{2}\right|^{2}\right) \Re\left[\lambda(t) \frac{f\left(z_{1}\right)}{z_{1} f^{\prime}\left(z_{1}\right)}\right]+\left|z_{2}\right|^{2}(1-\alpha) \Re \lambda(t) .
\end{aligned}
$$

Since $f$ is generalized spirallike with respect to $\lambda$ and $\alpha \in[0,1]$, we deduce that

$$
\Re\left\langle\left[D F_{\alpha}(z)\right]^{-1} A(t) F_{\alpha}(z), z\right\rangle>0, \text { a.e. } t \geq 0, \forall z \in B^{2} \backslash\{0\} .
$$

Therefore the relation (3.1) holds. On the other hand, since $|\lambda(t)| \leq b$, we deduce that $\|A(t)\| \leq b$ for $t \geq 0$. Moreover, since $\Re \lambda(t) \geq \eta>0$, we deduce that $\int_{0}^{\infty} m(A(t)) \overline{d t}=\infty$. Hence $F_{\alpha}$ is generalized spirallike with respect to $A$ by Theorem 3.5. This completes the proof.

We next give an example which shows the difference between spirallikeness and generalized spirallikeness with respect to a measurable diagonal operator.

Example 4.8. Let $\alpha \in(-\pi / 2, \pi / 2)$ and $f: U \rightarrow \mathbb{C}$ be given by

$$
f(z)=\frac{z}{(1-z)^{2 e^{-i \alpha} \cos \alpha}}, z \in U
$$

If $f$ is generalized spirallike with respect to a uniformly bounded measurable function $a:[0, \infty) \rightarrow \mathbb{C}$ with $\Re a(t) \geq \delta, t \geq 0$, for some $\delta>0$, then there exists a measurable function $r(t)$ such that $r(t)>0$ and $a(t)=r(t) e^{-i \alpha}$ for all $t \geq 0$.

Proof. Since

$$
f^{\prime}(z)=\frac{1+\left(2 e^{-i \alpha} \cos \alpha-1\right) z}{(1-z)^{2 e^{-i \alpha}} \cos \alpha+1},
$$

we deduce that the function

$$
g(z)=e^{i \alpha} \frac{z f^{\prime}(z)}{f(z)}
$$

maps 1 to $\infty,-1$ to $i \sin \alpha,-e^{2 i \alpha}$ to 0 and 0 to $e^{i \alpha}$. Thus, $g$ maps the unit disc onto the right half plane. This implies that $f$ is a spirallike function of type $\alpha$ and if $f$ is a generalized spirallike function with respect to $a(t)$, then $a(t)=r(t) e^{-i \alpha}$ with $r(t)>0$ for all $t$. 


\section{References}

[1] F. F. Bonsall and J. Duncan, "Numerical Ranges. II", Cambridge Univ. Press, 1973.

[2] E. A. Coddington and N. Levinson, "Theory of Ordinary Differential Equations", McGraw-Hill Book Co., New York-Toronto-London, 1955.

[3] Yu. L. DaleckiI and M.G. Krein, "Stability of Solutions of Differential Equations in a Banach Space", Translations of Mathematical Monographs, Vol. 43, American Mathematical Society, Providence, R.I., 1974.

[4] N. Dunford and J.T. Schwartz, "Linear Operators. I", Interscience Publ., Inc., New York, 1966.

[5] M. Elin, S. REICH and D. SHOIKHET, Complex dynamical systems and the geometry of domains in Banach spaces, Dissertationes Math. 427 (2004), 1-62.

[6] I. Graham, H. Hamada and G. Kohr, Parametric representation of univalent mappings in several complex variables, Canad. J. Math. 54 (2002), 324-351.

[7] I. Graham, H. Hamada, G. Kohr and M. Kohr, Parametric representation and asymptotic starlikeness in $\mathbb{C}^{n}$, Proc. Amer. Math. Soc. 136 (2008), 267-302.

[8] I. Graham, H. HamadA, G. KoHR and M. KoHR, Asymptotically spirallike mappings in several complex variables, J. Anal. Math. 105 (2008), 267-302.

[9] I. GRAHAm and G. KoHR, "Geometric Function Theory in One and Higher Dimensions", Marcel Dekker Inc., New York, 2003.

[10] I. Graham, G. KoHR and M. KoHR, Loewner chains and the Roper-Suffridge extension operator, J. Math. Anal. Appl. 247 (2000), 448-465.

[11] I. GRAHAM, G. KOHR and M. KoHR, Loewner chains and parametric representation in several complex variables, J. Math. Anal. Appl. 281 (2003), 425-438.

[12] K. Gurganus, $\Phi$-like holomorphic functions in $\mathbb{C}^{n}$ and Banach spaces, Trans. Amer. Math. Soc. 205 (1975), 389-406.

[13] K. E. Gustafson and D.K.M. Rao, "Numerical Range. The Field of Values of Linear Operators and Matrices", Springer-Verlag, New York, 1997.

[14] H. HAMADA and G. KOHR, Subordination chains and the growth theorem of spirallike mappings, Mathematica (Cluj) 42 (65) (2000), 153-161.

[15] H. HAMADA and G. KOHR, An estimate of the growth of spirallike mappings relatve to a diagonal matrix, Ann. Univ. Mariae Curie-Skłodowska, Sect. A. 55 (2001), 53-59.

[16] L. HARRIS, The numerical range of holomorphic functions in Banach spaces, Amer. J. Math. 93 (1971), 1005-1019.

[17] L. A. HARris, S. ReICH and D. ShoIKHET, Dissipative holomorphic functions, Bloch radii, and the Schwarz lemma, J. Anal. Math. 82 (2000), 221-232.

[18] G. KoHR, Using the method of Löwner chains to introduce some subclasses of biholomorphic mappings in $\mathbb{C}^{n}$, Rev. Roumaine Math. Pures Appl. 46 (2001), 743-760.

[19] J. A. PfaltzGRAFF, Subordination chains and univalence of holomorphic mappings in $\mathbb{C}^{n}$, Math. Ann. 210 (1974), 55-68.

[20] J. A. Pfaltzgraff and T.J. Suffridge, An extension theorem and linear invariant families generated by starlike maps, Ann. Univ. Mariae Curie-Skłodowska, Sect. A. 53 (1999), 193-207.

[21] C. Pommerenke, Über die subordination analytischer funktinonen, J. Reine Angew. Math. 218 (1965), 159-173.

[22] C. Pommerenke, "Univalent functions", Vandenhoeck \& Ruprecht, Göttingen, 1975.

[23] T. POREDA, On the univalent holomorphic maps of the unit polydisc in $\mathbb{C}^{n}$ which have the parametric representation, I-the geometrical properties, Ann. Univ. Mariae CurieSkłodowska, Sect. A. 41 (1987), 105-113.

[24] T. POREDA, On the univalent holomorphic maps of the unit polydisc in $\mathbb{C}^{n}$ which have the parametric representation, II-the necessary conditions and the sufficient conditions, Ann. Univ. Mariae Curie-Skłodowska, Sect. A. 41 (1987), 115-121. 
[25] T. PoredA, On the univalent subordination chains of holomorphic mappings in Banach spaces, Comment. Math. Prace Mat. 28 (1989), 295-304.

[26] T. POREDA, On generalized differential equations in Banach spaces, Dissertationes Math. 310 (1991), 1-50.

[27] S. Reich and D. Shoikhet, "Nonlinear Semigroups, Fixed Points, and Geometry of Domains in Banach Spaces", Imperial College Press, London, 2005.

[28] K. Roper and T. J. SUfFridge, Convex mappings on the unit ball of $\mathbb{C}^{n}$, J. Anal. Math. 65 (1995), 333-347.

[29] T. J. Suffridge, Starlike and convex maps in Banach spaces, Pacific J. Math. 46 (1973), $575-589$.

[30] T. J. SUFFRIDGE, Starlikeness, convexity and other geometric properties of holomorphic maps in higher dimensions, In: "Lecture Notes in Math.", Springer-Verlag 599 (1977), 146-159.

[31] K. YosidA, "Functional Analysis", Springer-Verlag, 1965.

\author{
Department of Mathematics \\ University of Toronto \\ Toronto, Ontario M5S 2E4, Canada \\ graham@math.toronto.edu \\ Faculty of Engineering \\ Kyushu Sangyo University \\ 3-1 Matsukadai 2-Chome, Higashi-ku \\ Fukuoka 813-8503, Japan \\ h.hamada@ip.kyusan-u.ac.jp \\ Faculty of Mathematics \\ and Computer Science \\ Babeş-Bolyai University \\ 1 M. Kogălniceanu Str. \\ 400084 Cluj-Napoca, Romania \\ gkohr@math.ubbcluj.ro \\ mkohr@math.ubbcluj.ro
}

